\title{
Molecular mechanisms underlying the positive role of treadmill training in locomotor recovery after spinal cord injury
}

\author{
Q Liu' ${ }^{1}$, B Zhang ${ }^{2}$, C Liu ${ }^{1}$ and D Zhao ${ }^{2}$
}

Objectives: This study aimed to investigate the molecular mechanisms underlying the positive role of treadmill training (TMT) in locomotor recovery.

Methods: GSE52763 microarray data were downloaded from GEO database, which was collected from the lumbar spinal cord samples of three groups of mice: mice subjected to contusive injury and killed 1 week after injury (I1), mice subjected to injury and killed 3 weeks after injury (I3), and mice subjected to injury and TMT beginning at week 1 and lasting until week 3 (T3). Differential expression analysis between 13 and 11 , between $T 3$ and $I 1$ and between $T 3$ and 13 were performed by $T$-test using R/LIMMA. Genes with $\| \log _{2} \mathrm{FC}$ (fold change) $\mid>0.58$ and $P$-value $<0.05$ were considered as differentially expressed genes (DEGs). Specific I3 vs II DEGs and T3 vs I1 DEGs were screened. Then TMT-induced specific DEGs were subject to functional and pathway enrichment analysis using DAVID online tool. Protein-protein interaction (PPI) analysis was also carried out using the STRING database.

Results: Finally, 82 upregulated DEGs and 297 downregulated DEGs were found specifically induced by TMT. Specific upregulated DEGs were mostly enriched in response to organic substance and morphogenesis-related events, and specific downregulated DEGs were related to positive regulation of transcription. ATP2A1, PRKACA, ITPR2 and so on had high connection degree in the PPI network of the specific upregulated DEGs; FOS, GSK3B and so on had high degrees in the PPI network of the specific downregulated DEGs. Conclusion: ATP2A1, C-FOS and GSK3B may have critical roles in the positive role of TMT in locomotor recovery. Spinal Cord (2017) 55, 441-446; doi:10.1038/sc.2016.134; published online 6 December 2016

\section{INTRODUCTION}

Spinal cord injury (SCI) refers to any injury to the spinal cord that can cause sensory and motor dysfunctions, with symptoms varying from pain to paralysis even to incontinence. Aside from lifetime deficits in locomotion, patients with SCI also have to bear huge financial burden. ${ }^{1}$ However, no satisfactory strategies for locomotor recovery have been presented so far.

Physical exercise has been applied for the recovery of motor function after SCI. Forssberg et al. ${ }^{2}$ first reported that the treadmill training (TMT) notably promoted the locomotor recovery in spinalized cats following SCI. Rossignol and collaborators have undertook many studies on TMT, especially the kinematics and the electromyographic activity during treadmill locomotion, mainly in cats. $^{3-5}$ In a systematic review of exercise applied to locomotor recovery, Battistuzzo et al. ${ }^{6}$ have demonstrated that TMT has superior performance in locomotor recovery as compared with other exercise types (body weight-supported TMT, voluntary wheel running and swimming) in different animal models of SCI, with positive outcomes in $100 \%$ of both rats and cats. However, the molecular mechanisms underlying the positive role of TMT in locomotor recovery have not been well elucidated.

Shin et al. ${ }^{7}$ investigated the mechanisms underlying the positive role of TMT in locomotor recovery using microarrays. Based on the genes differentially expressed at either 1 or 3 weeks after SCI, they found that the most robust upregulation was observed in genes related to immune or inflammation responses; more strikingly, the elevated expression of many inflammation-related genes was sustained in mice subjected to TMT and TMT upregulated the expression of neuroplasticity- and angiogenesis-related genes that were downregulated after SCI. ${ }^{7}$ Herein, using the microarray data submitted by Shin et al., ${ }^{7}$ we attempted to further investigate the molecular mechanisms underlying the positive role of TMT in locomotor recovery from a protein-protein interaction (PPI) perspective.

\section{MATERIALS AND METHODS}

\section{Source of microarray data}

The GSE52763 microarray data were downloaded from GEO database (GEO, http://www.ncbi.nlm.nih.gov/geo/). This data set was collected from lumbar spinal cord samples of three groups of mice: mice subjected to contusive injury and killed 1 week after injury $(n=4, \mathrm{I} 1)$, mice subjected to contusive injury and killed 3 weeks after injury $(n=4, \mathrm{I} 3)$ and mice subjected to contusive injury and TMT beginning at week 1 and lasting until week 3 after injury (TMT of 2-week duration) ( $n=4, \mathrm{~T} 3)$.

As described by Shin et al. ${ }^{7}$ in their original study, rats were first subjected to a dorsal laminectomy at the ninth thoracic vertebral level (T9) to expose the spinal cord and then contusion injury was performed by mechanical impact with a force of $200 \mathrm{kdyn}$. The rats were able to walk without auxiliary support at a slow speed $\left(5 \mathrm{~m} \mathrm{~min}^{-1}\right)$ prior to TMT. TMT was performed in the Flat Treadmill System (Model, IW-FT; IWOO Scientific Corporation, Seoul, Korea) for rats in combination with electric stimulation. Electric shocks of $1.2 \mathrm{~mA}$ were applied when an animal was not walking and displaced to the end

\footnotetext{
${ }^{1}$ The Second Hospital of Jilin University, Changchun, China and ${ }^{2}$ Department of Orthopedic, China-Japan Union Hospital, Jilin University, Changchun, China Correspondence: Dr D Zhao, Department of Orthopedic, China-Japan Union Hospital, Jilin University, 126 Xiantai Street, Changchun 130033, China. E-mail: zhaodxz@sina.com

Received 5 May 2016; revised 6 July 2016; accepted 20 July 2016; published online 6 December 2016
} 
of its runway, which were required several times on the first day of training, but were rarely applied after a few days. As locomotion was improved, the belt speed was gradually increased to $12 \mathrm{~m} \mathrm{~min}^{-1}$ during the first week and maintained at this level until the end of training. TMT was performed daily for 14 days, with each session lasting $30 \mathrm{~min}$. Locomotor recovery was assessed using Basso, Beattie and Bresnahan test. Please refer to the original study ${ }^{7}$ for details.

\section{Identification of differentially expressed genes (DEGs) and specific DEGs}

The downloaded data were first subject to background correction, quantile normalization and probe summarization by the RMA ( robust multiarray average) method $^{8}$ using R/Affy package. ${ }^{9}$ Next, differential expression analysis between $\mathrm{I} 3$ and I1, between $\mathrm{T} 3$ and $\mathrm{I} 1$ and between $\mathrm{T} 3$ and $\mathrm{I} 3$ was performed by $T$-test using the R/LIMMA (linear models for microarray data) package. ${ }^{10}$ Genes with $\mid \log _{2} \mathrm{FC}$ (fold change) $\mid>0.58$ and $P$-value $<0.05$ were considered as DEGs. DEGs between I3 and I1 (I3 vs I1 DEGs) and DEGs between T3 and I1 (T3 vs I1 DEGs) were further compared by screening the common ones. Specific I3 vs I1 DEGs or specific T3 vs I1 DEGs were further obtained via subtracting the common DEGs, respectively. The results were visualized using Venn plots. ${ }^{11}$

Functional annotation and pathway enrichment analysis of specific DEGs

DAVID (Database for Annotation, Visualization and Integrated Discovery) is an online tool integrating a comprehensive set of functional annotation tools that allows researchers to systematically extract the biological meaning from large gene/protein lists. ${ }^{12}$ The specific DEGs were submitted to DAVID for functional and pathway enrichment analysis based on Gene Ontology $(\mathrm{GO})^{13}$ and KEGG (Kyoto Encyclopedia of Genes and Genomes) pathway databases $(P<0.05){ }^{14}$

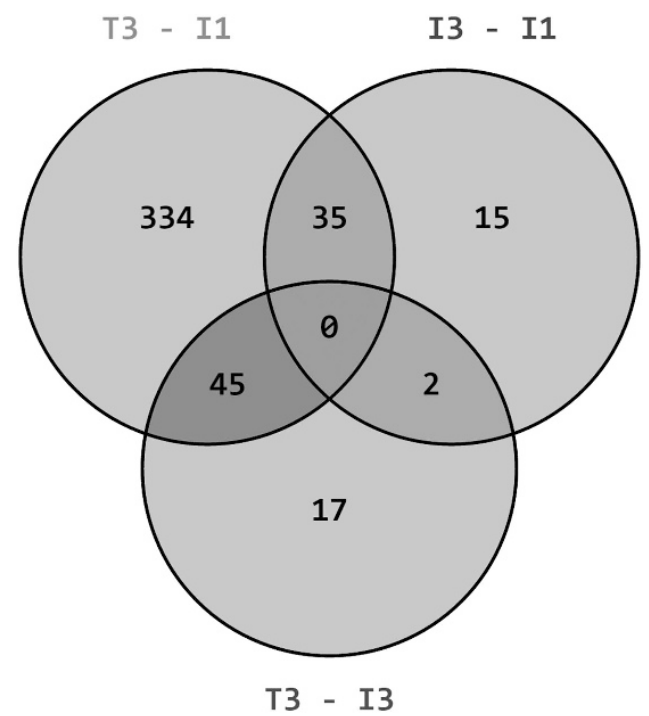

Figure 1 Comparison between T3 vs I1, I3 vs I1 and T3 vs I3 DEGs by Venn plot. T3 vs II DEGs represents DEGs between mice subjected to contusive injury and killed 1 week after injury (I1) and mice subjected to contusive injury and TMT beginning at week 1 and lasting until week 3 after injury (T3); 13 vs 11 DEGs represents DEGs between mice subjected to contusive injury and killed 3 weeks after injury (I3) and mice subjected to contusive injury and TMT beginning at week 1 and lasting until week 3 after injury (T3); T3 vs I3 DEGs represents DEGs between mice subjected to contusive injury and killed 3 weeks after injury $(n=4,13)$ and mice subjected to contusive injury and TMT beginning at week 1 and lasting until week 3 after injury (T3).

\section{Construction of PPI network}

To better understand the specific DEGs from an interactive respective, we used STRING (Search Tool for the Retrieval of Interacting Genes/Proteins) online tool to construct an interaction network of their protein products..$^{15}$ Combined score $>0.4$ was set as the cutoff for PPI. The connection degree of each protein in the network was also calculated. The resulting network was visualized using the Cytoscape software (version 2.8.3, National Institute of General Medical Sciences (NIGMS), Bethesda, MD, USA). ${ }^{16}$

\section{RESULTS}

TMT improved locomotor recovery after thoracic SCI

As described by Shin et al., ${ }^{7}$ rats exhibited enhanced locomotor recovery and higher-quality overground locomotion as they were subjected to TMT. The locomotor improvement induced by TMT was statistically significant compared with control rats.

\section{Screening of DEGs and specific DEGs}

Finally, 16 upregulated DEGs and 36 downregulated DEGs were identified between the I3 and I1 groups (I3vs I1 DEGs), 89 upregulated and 325 downregulated between the I1 and T3 groups (T3vs I1 DEGs) and 12 upregulated and 52 downregulated between the T3 and I3 groups (T3 vs I3 DEGs), indicating that TMT has induced alteration in the expression of a substantially larger number of genes. The Venn plot revealed 35 common DEGs between T3 vs I1 DEGs and I3 vs I1 DEGs, among which 7 were upregulated and 28 were downregulated (Figure 1). Finally, 82 upregulated T3 vs I1 DEGs and 297 downregulated T3 vs I1 DEGs were found specific, and 9 upregulated I3 vs I1 DEGs and 8 downregulated I3 vs I1 DEGs were found specific (Table 1).

GO functional annotation and KEGG enrichment analysis of the specific upregulated genes and downregulated regulated genes According to GO functional annotation, the specific upregulated DEGs were mostly enriched in biological processes related to response to organic substance (vitamin, vitamin A, lipopolyaccharide, nutrient) and endogenous/extracellular stimulus, as well as morphogenesisrelated events (Table 2). According to the KEGG pathway enrichment analysis, three DEGs IGF1, PRKACA and ITPR2 were significantly enriched in the pathway oocyte meiosis (rno04114).

Meanwhile, GO functional annotation revealed that the specific downregulated T3 vs I1 DEGs were related to positive regulation of transcription (Table 3). And some of the downregulated DEGs were enriched in two KEGG pathways: rno05200:Pathways in cancer (FOS, MAX, BCR, PTGS2, MMP9, GSK3B, CBL and RUNX1T1) and rno04660:T-cell receptor signaling pathway (FOS, GSK3B, CBL, MAP3K8 and NFAT5), according to the pathway enrichment analysis.

\section{Construction of PPI network and module analysis}

The PPI network of the specific upregulated T3 vs I1 DEGs includes 31 interaction pairs (Figure 2), and the top eight proteins with the highest connection degree were ATP2A1 (degree=6),

Table 1 Specific differential genes in each group

\begin{tabular}{lccc}
\hline & Up & Down & Total \\
\hline T3 vs I1 DEGs & 82 & 297 & 379 \\
I3 vs I1 DEGs & 9 & 8 & 17 \\
Common & 7 & 28 & 35 \\
\hline
\end{tabular}

Abbreviation: DEG, differentially expressed gene. 
Table 2 Gene Ontology (GO) analysis of the specific upregulated differentially expressed genes

\begin{tabular}{|c|c|c|c|}
\hline GO biological process term & Count & P-value & Differentially expressed genes \\
\hline GO:0048729-tissue morphogenesis & 5 & $3.76 \mathrm{E}-04$ & NOTCH2, CD44, RXRA, IGF1, PRKACA \\
\hline GO:0031099-regeneration & 4 & $7.64 \mathrm{E}-04$ & NOTCH3, NOTCH2, RXRA, IGF1 \\
\hline GO:0030850_prostate gland development & 3 & $1.51 \mathrm{E}-03$ & CD44, RXRA, IGF1 \\
\hline GO:0001655—urogenital system development & 4 & $1.72 \mathrm{E}-03$ & CD44, RXRA, MYO1E, IGF1 \\
\hline G0:0042060—wound healing & 4 & 2.40E-03 & NOTCH3, NOTCH2, CD44, IGF1 \\
\hline GO:0014070-response to organic cyclic substance & 4 & 4.13E-03 & CD44, CYP1A1, RXRA, IGF1 \\
\hline
\end{tabular}

Table 3 Gene Ontology analysis of the specific downregulated differentially expressed genes

\begin{tabular}{|c|c|c|c|}
\hline $\begin{array}{l}\text { GO:0045944-positive regulation of transcription from RNA } \\
\text { polymerase II promoter }\end{array}$ & 14 & 1.97E-05 & $\begin{array}{l}\text { CEBPB, KLF13, NFIX, SIX4, MED21, HOXD10, FOS, ZFP462, BCL11B, NFAT5, } \\
\text { TCF4, KLF4, NFIB, MED1 }\end{array}$ \\
\hline GO:0051254-positive regulation of RNA metabolic process & 15 & $3.02 \mathrm{E}-05$ & $\begin{array}{l}\text { CEBPB, KLF13, ILF3, NFIX, SIX4, MED21, HOXD10, FOS, ZFP462, BCL11B, } \\
\text { NFAT5, TCF4, KLF4, NFIB, MED1 }\end{array}$ \\
\hline G0:0010628-positive regulation of gene expression & 16 & $5.12 \mathrm{E}-05$ & $\begin{array}{l}\text { CEBPB, KLF13, ILF3, NFIX, SIX4, MED21, HOXD10, FOS, ZFP462, BCL11B, } \\
\text { NFAT5, TCF4, CCNA2, KLF4, MED1, NFIB }\end{array}$ \\
\hline $\begin{array}{l}\text { GO:0010557-positive regulation of macromolecule biosyn- } \\
\text { thetic process }\end{array}$ & 17 & 6.77E-05 & $\begin{array}{l}\text { UTS2, CEBPB, KLF13, ILF3, NFIX, SIX4, MED21, HOXD10, FOS, ZFP462, BCL11B } \\
\text { NFAT5, TCF4, CCNA2, KLF4, MED1, NFIB }\end{array}$ \\
\hline $\begin{array}{l}\text { GO:0006357-regulation of transcription from RNA polymer- } \\
\text { ase II promoter }\end{array}$ & 16 & $1.55 \mathrm{E}-04$ & $\begin{array}{l}\text { CEBPB, KLF13, NFIX, SIX4, MED21, MED13L, HOXD10, FOS, ZFP462, BCL11B, } \\
\text { NFAT5, TCF4, NCOR1, KLF4, MED1, NFIB }\end{array}$ \\
\hline G0:0006355-regulation of transcription, DNA dependent & 24 & $1.75 \mathrm{E}-04$ & $\begin{array}{l}\text { MEF2A, CEBPB, ARID4A, KLF13, RUNX1T1, ILF3, NFIX, SIX4, MED21, MED13L, } \\
\text { HOXD10, MAX, FOS, ZFP462, TRPS1, GSK3B, BCL11B, NFAT5, TCF4, NCOR1, } \\
\text { KLF4, MLLT3, MED1, NFIB }\end{array}$ \\
\hline
\end{tabular}

Abbreviation: GO, Gene Ontology.

MYOZ1 (degree $=6)$, TNNI2 (degree $=6)$, PRKACA (degree $=5)$, ACTN3 (degree =5), CKM (degree=4), MYH4 (degree=5) and ITPR2 (degree $=2)$.

The PPI network of the specific downregulated T3 vs I1 DEGs comprises 335 interaction pairs (Figure 3), and the top eight proteins with the highest connection degree to other proteins were FOS $($ degree $=22)$, ASHLL $($ degree $=21)$, MEF2A (degree $=20)$, GSK3B $($ degree $=13)$, DDX6 $($ degree $=22)$, CSNK2A1 $($ degree $=20)$, ZC3H13 $($ degree $=14)$ and ESF1 (degree $=13)$.

\section{DISCUSSION}

Using the microarray data submitted by Shin et al., ${ }^{7}$ we focused on genes whose differential expression was specifically induced by TMT and attempted to elucidate their roles in TMT-induced locomotor recovery from a PPI perspective in the present study. It was found that a notably larger number of genes showed differential expression in mice subject to TMT compared with those that were not subjected to; furthermore, the upregulated specific DEGs in mice undergoing TMT are functionally related in response to organic substance (vitamin, vitamin A, lipopolyaccharide, nutrient) and endogenous/extracellular stimulus, as well as morphogenesisrelated events, and the specific downregulated T3 vs I1 DEGs were mainly related to positive regulation of transcription.

In the present study, the upregulated specific DEGs in mice undergoing TMT are functionally related in response to organic substance (vitamin, vitamin A, lipopolyaccharide, nutrient) and endogenous/extracellular stimulus, as well as morphogenesis-related events. This is consistent with a previous finding that the expression of neuroplasticity- and angiogenesis-related genes was upregulated by $\mathrm{TMT}^{7}$ implying that the positive role of TMT in locomotor recovery may be related to its beneficial effect on neuronal regeneration. Among these genes, ATP2A1 encodes an intracellular calcium pump ATPase 1, which is located in the sarcoplasmic or endoplasmic reticula of muscle cells. This protein is involved in muscular excitation and contraction, and mutation in human ATP2A1 gene has been reported to cause Brody disease, a rare disorder characterized by impaired 


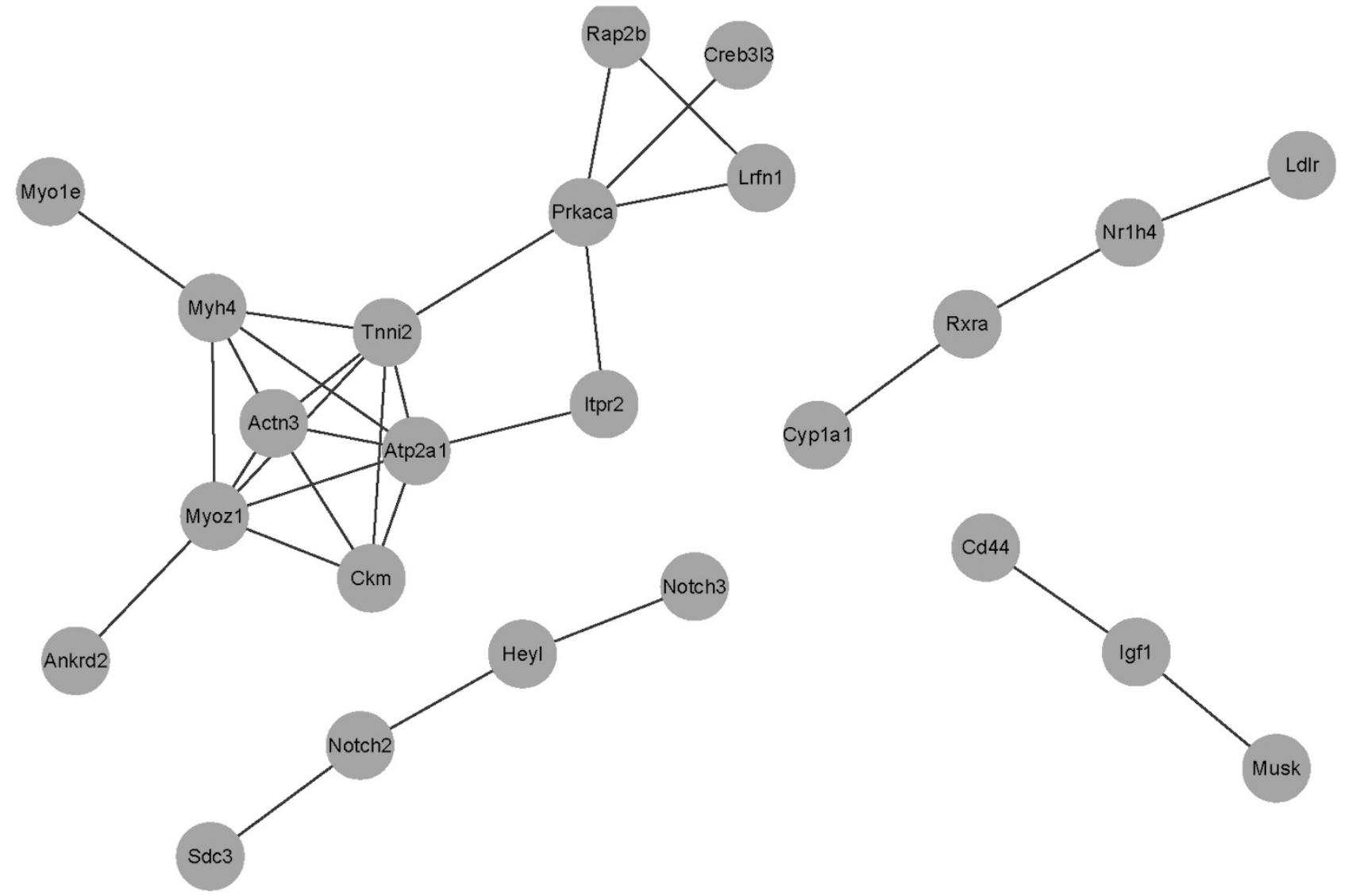

Figure 2 The PPI network of the specific upregulated T3 vs II DEGs. T3 vs I1 DEGs represents DEGs between mice subjected to contusive injury and killed 1 week after injury (I1) and mice subjected to contusive injury and TMT beginning at week 1 and lasting until week 3 after injury (T3). A full color version of this figure is available at the Spinal Cord journal online.

skeletal muscle relaxation. ${ }^{17}$ This gene was expressed in neural stem cells. $^{18}$ Its upregulation in mice subject to TMT indicates that it may have a positive role in locomotor recovery following TMT. Additionally, we also observed the upregulated expression in another two genes PRKACA and ITPR2. PRKACA encodes the catalytic subunit $\alpha$ of a cAMP-dependent protein kinase A that can activate substrate via phosphorylation at serine or threonine residue. ${ }^{19}$ Meyer et $a .^{20}$ have reported a noted increase in its expression within the anterior horn of murine lumbar spinal cord and presented that this gene is closely associated with motor neuron disease. ITPR2 encodes inositol 1,4,5-trisphosphate receptor, type 2, a calcium channel expressed in the endoplasmic reticulum, ${ }^{21}$ which is one of the main regulators of intracellular calcium concentrations in neurons. ${ }^{22}$ The overexpression of this gene was said to be responsible for the apoptosis of motor neurons. ${ }^{23,24}$ The upregulation of these two proteins may suggest that the apoptosis of motor neurons continues in mice with TMT, which seemingly conforms to the finding that the inflammation was not weakened in mice subjected to TMT. ${ }^{7}$

Among the specific downregulated TMT-induced genes, FOS encodes C-FOS transcription factor protein that can form heterodimer activator protein 1 with C-JUN (a JUN family of transcription factors) $)^{25,26}$ and activator protein 1 regulates gene expression in a wide range of biological events. ${ }^{27}$ C-FOS has been suggested as a marker for neuronal activity. ${ }^{28,29}$ Previous studies have consistently reported the increase in these two proteins in the spinal dorsal horn shortly (around a week) following SCI. ${ }^{30,31}$ However, the dynamics and site of its long-term expression remain controversial, ${ }^{32-34}$ indicating a complex role of this protein after SCI. Here mice subject to TMT showed a decrease in C-FOS level 3 weeks after SCI, supporting the view that this immediate early protein has been downregulated after 2 weeks of exercise; although differential expression was not observed in C-JUN. Another downregulated gene GSK3B encodes GSK3 $\beta$, one of the two glycogen synthase kinase-3 isoforms which inhibits axon growth. ${ }^{35}$ However, the role of this kinase in neural development may be complex. Inhibition of GSK3 $\beta$ can promote the regeneration of CNS axons in SCI animal model ${ }^{36}$ while robust inhibition of this enzyme may impair axon regeneration. ${ }^{37}$ Furthermore, Parkitna et al. ${ }^{38}$ have reported that intrathecal injection of GSK3 $\beta$ inhibitor can restore the analgesic effect of morphine in morphine-tolerant rats, thus GSK3 $\beta$ inhibition is supposed to alleviate neuropathic pain. ${ }^{39}$ Here GSK3 $\beta$ downregulation was observed in mice subject to TMT, implying that TMT can reduce GSK3 $\beta$ expression, which may not only benefit neuronal regeneration but also ameliorate the neuropathic pain. In the present study, both C-FOS and GSK3 $\beta$ were significantly enriched in the two pathways: pathways in cancer and T-cell receptor signaling pathway. This suggests that TMT may benefit locomotor recovery via reducing the expression of some key genes involved in these two pathways.

Taken together, the mechanisms underlying TMT's positive role in locomotor recovery may be complex. TMT seems to benefit neuronal regeneration by regulating related genes, especially ATP2A1, C-FOS and GSK3B; the proteins encoded by these genes were speculated to 


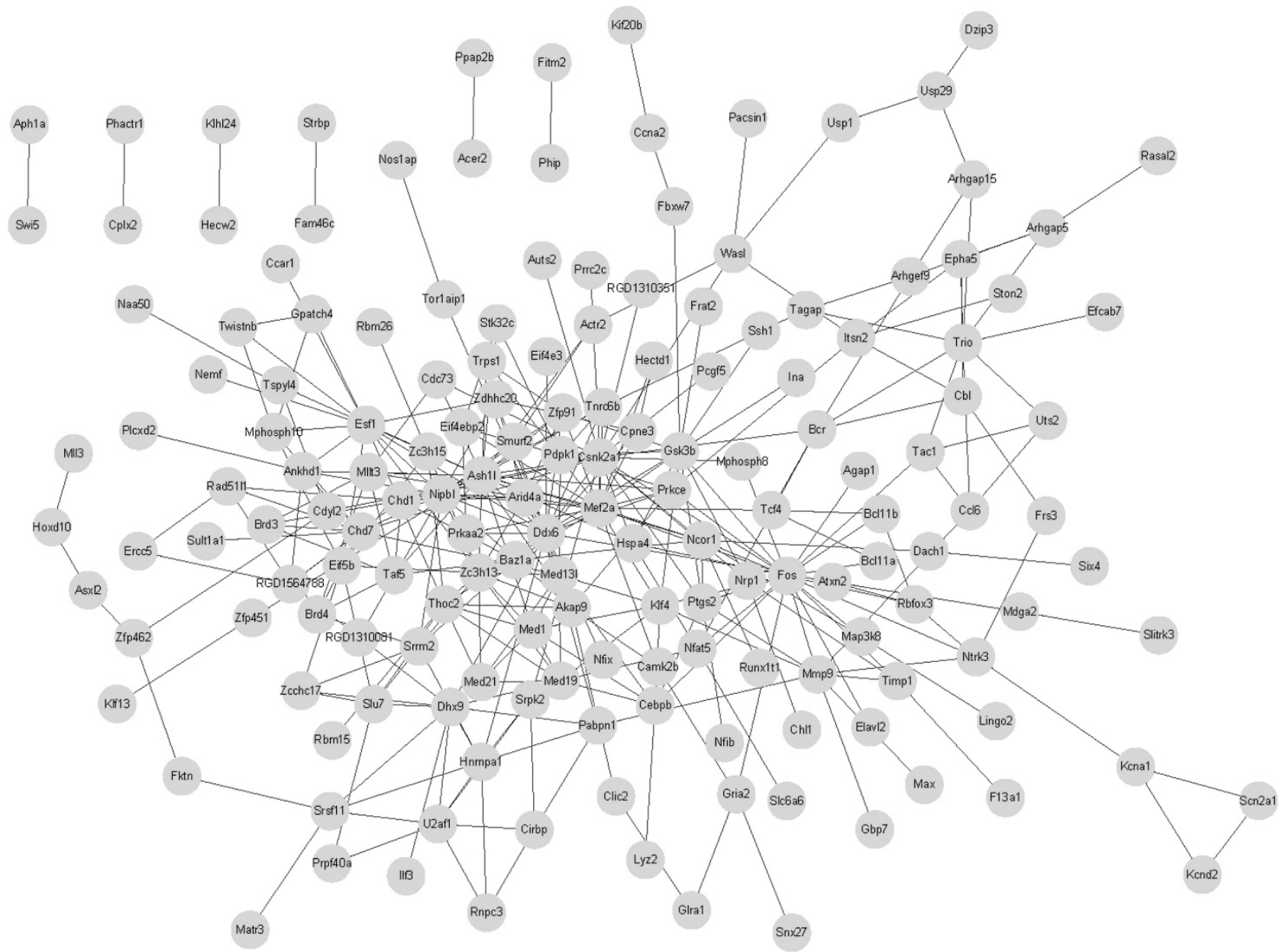

Figure 3 The PPI network of the specific downregulated T3 vs I1 DEGs. T3 vs I1 DEGs represents DEGs between mice subjected to contusive injury and killed 1 week after injury (I1) and mice subjected to contusive injury and TMT beginning at week 1 and lasting until week 3 after injury (T3). A full color version of this figure is available at the Spinal Cord journal online.

interact with many other specific proteins according to the PPI analysis, indicating their critical roles in locomotor recovery following TMT. Nevertheless, TMT seems not to change some adverse events following SCI in mice, such as apoptosis of motor neurons. However, our findings need to be validated by further experimental proofs and thus should be taken prudently at present.

\section{DATA ARCHIVING}

There were no data to deposit.

\section{CONFLICT OF INTEREST}

The authors declare no conflict of interest.

1 Oliveri RS, Bello S, Biering-Sørensen F. Mesenchymal stem cells improve locomotor recovery in traumatic spinal cord injury: systematic review with meta-analyses of rat models. Neurobiol Dis 2014; 62: 338-353.

2 Forssberg $\mathrm{H}$, Grillner S, Halbertsma J. The locomotion of the low spinal cat I. Coordination within a hindlimb. Acta physiol Scand 1980; 108: 269-281.

3 Belanger M, Drew T, Provencher J, Rossignol S. A comparison of treadmill locomotion in adult cats before and after spinal transection. J Neurophysiol 1996; 76 471-491.
4 Leblond H, L'Espérance M, Orsal D, Rossignol S. Treadmill locomotion in the intact and spinal mouse. J Neurosci 2003; 23: 11411-11419.

5 Barbeau H, Rossignol S. Recovery of locomotion after chronic spinalization in the adult cat. Brain Res 1987; 412: 84-95.

6 Battistuzzo CR, Callister RJ, Callister R, Galea MP. A systematic review of exercise training to promote locomotor recovery in animal models of spinal cord injury. J Neurotrauma 2012; 29: 1600-1613.

7 Shin HY, Kim H, Kwon MJ, Hwang DH, Lee K, Kim BG. Molecular and cellular changes in the lumbar spinal cord following thoracic injury: regulation by treadmill locomotor training. PLOS ONE 2014; 9: e88215.

8 Irizarry RA, Hobbs B, Collin F, Beazer-Barclay YD, Antonellis KJ, Scherf U et al. Exploration, normalization, and summaries of high density oligonucleotide array probe level data. Biostatistics 2003; 4: 249-264.

9 Gautier L, Cope L, Bolstad BM, Irizarry RA. Affy-analysis of Affymetrix GeneChip data at the probe level. Bioinformatics 2004; 20: 307-315.

10 Diboun I, Wernisch L, Orengo CA, Koltzenburg M. Microarray analysis after RNA amplification can detect pronounced differences in gene expression using LIMMA. BMC Genomics 2006; 7: 252

11 Oliveros JC. An interactive tool for comparing lists with Venn Diagrams. VENNY, 2007. http://bioinfogp.cnb.csic.es/tools/venny/index.html.

12 Huang DW, Sherman BT, Lempicki RA. Systematic and integrative analysis of large gene lists using DAVID bioinformatics resources. Nat Protoc 2008; 4: 44-57.

13 Ashburner M, Ball CA, Blake JA, Botstein D, Butler H, Cherry JM et al. Gene Ontology: tool for the unification of biology. Nat Genet 2000; 25: 25-29.

14 Aoki-Kinoshita KF, Kanehisa M. Gene annotation and pathway mapping in KEGG. Methods Mol Biol. 2007; 396: 71-91.

15 Franceschini A, Szklarczyk D, Frankild S, Kuhn M, Simonovic M, Roth A et al. STRING v9. 1: protein-protein interaction networks, with increased coverage and integration. Nucleic Acids Res 2013; 41: D808-D815. 
16 Shannon P, Markiel A, Ozier O, Baliga NS, Wang JT, Ramage D et al. Cytoscape: a software environment for integrated models of biomolecular interaction networks. Genome Res 2003; 13: 2498-2504.

17 Odermatt A, Taschner PE, Khanna VK, Busch HF, Karpati G, Jablecki CK et al. Mutations in the gene-encoding SERCA1, the fast-twitch skeletal muscle sarcoplasmic reticulum Ca2\&plus; ATPase, are associated with Brody disease. Nat Genet 1996; 14: 191-194.

18 Cai J, Cheng A, Luo Y, Lu C, Mattson MP, Rao MS et al. Membrane properties of rat embryonic multipotent neural stem cells. J Neurochem 2004; 88: 212-226.

19 Banday AR, Azim S, Hussain MA, Nehar S, Tabish M. Computational prediction and characterisation of ubiquitously expressed new splice variant of Prkaca gene in mouse. Cell Biol Int 2013; 37: 687-693.

20 Meyer MA. Identification of 17 highly expressed genes within mouse lumbar spinal cord anterior horn region from an in-situ hybridization atlas of 3430 genes: implications for motor neuron disease. Neurol Int 2014; 6: 5367.

21 Yamamoto-Hino M, Sugiyama T, Hikichi K, Mattei M, Hasegawa K, Sekine S et al. Cloning and characterization of human type 2 and type 3 inositol 1, 4, 5-trisphosphate receptors. Receptors Channels 1993; 2: 9-22.

22 Mikoshiba K. Inositol 1, 4, 5-trisphosphate (IP3) receptors and their role in neuronal cell function. J Neurochem 2006; 97: 1627-1633.

23 Van Den Bosch L, Verhoeven K, De Smedt H, Wuytack F, Missiaen L, Robberecht W. Calcium handling proteins in isolated spinal motoneurons. Life Sci 1999; 65 1597-1606.

24 Cleveland DW, Rothstein JD. From Charcot to Lou Gehrig: deciphering selective motor neuron death in ALS. Nat Rev Neurosci 2001; 2: 806-819.

25 Kerppola T, Curran T. Selective DNA bending by a variety of bZIP proteins. Mol Cell Biol 1993; 13: 5479-5489.

26 Karin M, Liu Z-g, Zandi E. AP-1 function and regulation. Curr Opin Cell Biol 1997; 9: 240-246.

27 Shaulian E, Karin M. AP-1 as a regulator of cell life and death. Nat Cell Biol 2002; 4: E131-E136.
28 Dragunow M, Faull R. The use of $\mathrm{c}$-fos as a metabolic marker in neuronal pathway tracing. J Neurosci Methods 1989; 29: 261-265.

29 Bullitt E. Expression of C-fos-like protein as a marker for neuronal activity following noxious stimulation in the rat. J Comp Neurol 1990; 296: 517-530.

30 Munglani R, Hunt SP. Proto-oncogenes: basic concepts and stimulation induced changes in the spinal cord. Prog Brain Res 1995; 104: 283-298.

31 Morgan JI, Curran T. Stimulus-transcription coupling in the nervous system: involvement of the inducible proto-oncogenes fos and jun. Annu Rev Neurosci 1991; 14: 421-451.

32 Hudspith M, Harrisson S, Smith G, Bountra C, Elliot P, Birch P et al. Effect of postinjury NMDA antagonist treatment on long-term Fos expression and hyperalgesia in a model of chronic neuropathic pain. Brain Res 1999; 822: 220-227.

33 Catheline G, Le Guen S, Honoré P, Besson J-M. Are there long-term changes in the basal or evoked Fos expression in the dorsal horn of the spinal cord of the mononeuropathic rat? Pain 1999; 80: 347-357.

34 Kajander KC, Madsen AM, Iadarola MJ, Draisci G, Wakisaka S. Fos-like immunoreactivity increases in the lumbar spinal cord following a chronic constriction injury to the sciatic nerve of rat. Neurosci Lett 1996; 206: 9-12.

35 Seira O, Gavín R, Gil V, Llorens F, Rangel A, Soriano E et al. Neurites regrowth of cortical neurons by GSK3 $\beta$ inhibition independently of Nogo receptor 1. J Neurochem 2010; 113: 1644-1658.

36 Dill J, Wang H, Zhou F, Li S. Inactivation of glycogen synthase kinase 3 promotes axonal growth and recovery in the CNS. J Neurosci 2008; 28: 8914-8928.

37 Alabed YZ, Pool M, Tone SO, Sutherland C, Fournier AE. GSK3 $\beta$ regulates myelindependent axon outgrowth inhibition through CRMP4. J Neurosci 2010; 30: 5635-5643.

38 Parkitna JR, Obara I, Wawrzczak-Bargiela A, Makuch W, Przewlocka B, Przewlocki R. Effects of glycogen synthase kinase $3 \beta$ and cyclin-dependent kinase 5 inhibitors on morphine-induced analgesia and tolerance in rats. J Pharmacol Exp Ther 2006; 319: 832-839.

39 Yang M, Li J, So K, Chen J, Cheng W, Wu J et al. Efficacy and safety of lithium carbonate treatment of chronic spinal cord injuries: a double-blind, randomized, placebo-controlled clinical trial. Spinal Cord 2012; 50: 141-146. 